\title{
ON AN ASYMPTOTIC PROPERTY OF A VOLTERRA INTEGRAL EQUATION
}

\author{
A. F. $I Z E^{1}$
}

Abstract. It is proved that if $q(t-s)$ is bounded and $f(t, x)$ is "small," the solutions of the integral equation $x(t)=a(t)$ $+\int_{0}^{t} q(t-s) f(s, x(s)) d s$ satisfies the conditions $x(t)=h(t)+\rho(t) a(t)$, $\lim _{t \rightarrow \infty} a(t)=a$ constant where $\rho(t)$ is a nonsingular diagonal matrix chosen in such a way that $\rho(t) h(t)$ is bounded. The results are extended to the more general integral equation $x(t)=h(t)$ $+\int_{0}^{t} F(t, s, x(s)) d s$ and contain, in particular, some results on the boundedness, asymptotic behavior and existence of nonoscillatory solution of differential equations.

Consider the system of Volterra integral equations

$$
x(t)=h(t)+\int_{0}^{t} q(t-\tau) f(\tau, x(\tau)) d \tau+\int_{0}^{t} q(t-\tau) g(\tau) d \tau
$$

where $h=\left(h_{1}, h_{2}, \cdots, h_{n}\right), f=\left(f_{1}, f_{2}, \cdots, f_{n}\right), g=\left(g_{1}, g_{2}, \cdots, g_{n}\right)$ are column vectors in $E^{n}, q=\left(q_{i j}\right)$ is an $n \times n$ matrix and $E^{n}$ is Euclidean $n$-dimensional space. In what follows $\lim _{t \rightarrow \infty}$ means limit almost everywhere.

We assume the hypotheses

$\left(\mathrm{H}_{1}\right)|q(t)|$ and $|g(t)| \in L(0, C], 0<C<\infty$,

$\left(\mathrm{H}_{2}\right) h(t)$ is continuous for $0 \leqq t<\infty$,

$\left(\mathrm{H}_{3}\right) f(t, x)$ is continuous for $0 \leqq t<\infty,|x|<\infty$.

These conditions guarantee the local existence of continuous solutions and continuability of each solution so long as they remain bounded [6, p. 324]. We show here that if $f, g$ and $q$ satisfy some additional hypotheses then the solutions of (1) satsify the condition $x_{i}(t)=h_{i}(t)+a_{i}(t) \rho_{i}(t)$ with $\lim _{t \rightarrow \infty} a_{i}(t)=a_{i}$ or $a_{i}(t)$ bounded, where $\rho_{i}(t)>0$ is chosen in such a way that $h_{i}(t) / \rho_{i}(t)$ is bounded. Some similar results were obtained by Strauss [8] under different hypotheses. We need the following lemma.

LEMMa 1. Let $E$ be a measurable set of points of any number of dimensions of finite or infinite measure and let $f(t, s)$ be summable in $E$ for

Presented to the Society, April 17, 1970; received by the editors December 2, 1969.

AMS 1969 subject classifications. Primary 4135, 4530; Secondary 3450.

Key words and phrases. Volterra integral equations, asymptotic properties, measurable set, summable, almost all, global existence, kernel, asymptotic behavior asymptotic equilibrium.

1 This research was partially supported by Conselho Nacional de Pesquisa, Brasil. 
values of $t$ in $[0, \infty)$. Assume that there exists a summable nonnegative function $\phi(s)$ such that $|f(t, s)| \leqq \phi(s)$ for almost all values of $s$ in $E$ and all values of $t$ in $\left(t_{0}, \infty\right)$. Then if $\lim _{t \rightarrow \infty} f(t, s)$ exists for all (or almost all) values of $\sin E$ we have

$$
\lim _{t \rightarrow \infty} \int_{E} f(t, s) d s=\int_{E} \lim _{t \rightarrow \infty} f(t, s) d s .
$$

For a proof of Lemma 1, see [4, p. 322]. The following lemma which is proved in a more general form in $[6$, p. 326] gives conditions for the global existence of solutions of (1).

LEMMA 2. Let $\omega(t, r)$ be continuous in $(t, r)$ for $0 \leqq t<\infty, 0 \leqq r<\infty$, and nondecreasing in $r$ for each fixed $t$. For some $b<\infty$ let $r_{M}$ be the maximum solution of

$$
\dot{r}=K \omega(t, r), \quad 0 \leqq t<b, \quad{ }^{\prime}=d / d t, \quad r(0)=K,
$$

and suppose that $|h(t)| \leqq K,|q(t)| \leqq K$ and $|f(t, x)| \leqq \omega(t,|x|)$, $0 \leqq t<\infty,|x|<\infty$. Then if $\phi$ is a solution of (1) $\phi$ can be continued to the right as far as $r_{M}$ exists and

$$
|\phi(t)| \leqq r_{M}(t), \quad 0 \leqq t<b .
$$

In particular, if $r_{M}(t)$ exists for $0 \leqq t<\infty$ one has global existence for solutions of (1).

LEMMA 3. Let $a_{i} \geqq 0, b_{i} \geqq 0, r_{i} \geqq 0$ and $r=\max _{i} r_{i}, i=1,2, \cdots, n$, if $b_{i}>1$ for some $i$ then

$$
\sum_{i=1}^{n} a_{i} b_{i}^{r_{1}} \leqq\left[\sum_{i=1}^{n} a_{i}\right]\left[\sum_{i=1}^{n} b_{i}\right]^{r} .
$$

Let $\rho_{i}(t)>0, i=1,2, \cdots, n$, be continuous functions for $0 \leqq t<\infty$ in such a way that $h_{i}(t) / \rho_{i}(t), i=1,2, \cdots, n$, are bounded for $0 \leqq t<\infty$.

We assume also the hypotheses

$\left(H_{4}\right)\left|f_{i}(t, x)\right| \leqq \sum_{j=1}^{n} \epsilon_{i j}(t)\left|x_{j}\right| r_{i}, i=1,2, \cdots, n$, where $\epsilon_{i j}(t)$ is nonnegative, $r_{j}>0, j=1,2, \cdots, n,|x|<H \leqq \infty$ and

$$
\int^{\infty} \epsilon_{i j}(t)\left[\rho_{j}(t)\right]^{r}\left[\rho_{i}(t)\right]^{-1} d t<\infty .
$$

$\left(\mathrm{H}_{6}\right) \int^{\infty}\left|g_{i}(t)\right|\left[\rho_{i}(t)\right]^{-1} d t<\infty$.

$\left(\mathrm{H}_{6}\right) q_{i j}(t-\tau) \rho_{j}(\tau) / \rho_{i}(t)$ are bounded for $0 \leqq \tau \leqq t<\infty, i, j=1$, $2, \cdots, n$.

$\left(\mathrm{H}_{6}^{\prime}\right) q_{i j}(t-\tau) \rho_{j}(\tau) / \rho_{i}(t)$ are bounded for $0 \leqq \tau \leqq t<\infty$ and $\lim _{t \rightarrow \infty}\left(q_{i j}(t-\tau) / \rho_{i}(t)\right)$ exists for almost all values of $\tau$. 
THEOREM 1. Assume with respect to equation (1) the hypotheses $\left(\mathrm{H}_{4}\right)$, $\left(\mathrm{H}_{5}\right)$ and $\left(\mathrm{H}_{6}\right)$ and let $r$ be the maximum of the $r_{i}, i=1,2, \cdots, n$. Then

A. If $r>1$ there exists $c>0$ depending on $r$ and $\epsilon_{i j}, i, j=1,2, \cdots, n$, such that $\left|h_{i}(t) / \rho_{i}(t)\right| \leqq c, i=1,2, \cdots, n$, implies that the solutions of (1) exist in $[0, \infty)$ and satisfy the condition

$$
x_{i}(t)=h_{i}(t)+a_{i}(t) \rho_{i}(t)
$$

where $a_{i}(t)$ is bounded. If instead of condition $\left(\mathrm{H}_{6}\right),(1)$ satisfies $\left(\mathrm{H}_{6}^{\prime}\right)$ then $\lim _{t \rightarrow \infty} a_{i}(t)=a_{i}$ constant. Furthermore, if $\lim _{t \rightarrow \infty}\left(h_{i}(t) / \rho_{i}(t)\right)=h_{i}$ constant

$$
\lim _{t \rightarrow \infty} \frac{x_{i}(t)}{\rho_{i}(t)}=b_{i} \text { constant }
$$

B. If $r \leqq 1, c$ can be chosen arbitrarily.

ProOF.

$$
x_{i}(t)=h_{i}(t)+\int_{0}^{t} \sum_{r=1}^{n} q_{i r}(t-s) f_{r}(s, x(s)) d s+\int_{0}^{t} \sum_{j=1}^{n} q_{i j}(t-s) g_{j}(s) d s
$$

or

$$
\begin{aligned}
\frac{\left|x_{i}(t)\right|}{\rho_{i}(t)} \leqq & k_{i}+\int_{0}^{t} \sum_{r=1}^{n} \frac{\left|q_{i r}(t-s)\right| \rho_{r}(s)}{\rho_{i}(t)} \sum_{j=1}^{n} \epsilon_{r j}(s)\left[\rho_{j}(s)\right]^{r_{j}} \rho_{r}^{-1}(s) \frac{\left|x_{j}(s)\right|^{r_{j}} d s}{\left[\rho_{j}(s)\right]^{r_{j}}} \\
& +\int_{0}^{t} \sum_{j=1}^{n} \frac{\left|q_{i j}(t-s)\right| \rho_{j}(s) \rho_{j}(s)^{-1} \mid}{\rho_{i}(t)}\left|g_{j}(s)\right| d s \\
\leqq & k_{i}+\int_{0}^{t} \sum_{r=1}^{n} c_{r} \sum_{j=1}^{n} \epsilon_{r j}(s)\left[\rho_{j}(s)\right]^{r_{j}} \rho_{r}(s)^{-1} \frac{\left|x_{j}(s)\right|^{r_{j}}}{\left[\rho_{j}(s)\right]^{r_{j}}} d s \\
& +\int_{0}^{t} \sum_{j=1}^{n} c_{j}\left|g_{j}(s) \rho_{j}(s)^{-1}\right| d s \\
\leqq & K_{i}+k \int_{0}^{t}\left(\sum_{r j} \epsilon_{r j}(s)\left[\rho_{j}(s)\right]^{r_{j}} \rho_{r}(s)^{-1}\right) \sum_{i=1}^{n} \frac{\left|x_{i}(s)\right|^{r_{i}}}{\left[\rho_{i}(s)\right]^{r_{i}}} d s,
\end{aligned}
$$

where $K_{i}=k_{i}+\int_{0}^{\infty} \sum_{j=1}^{n} c_{j}\left|g_{j}(s)\right| \rho_{j}(s)^{-1} \mid d s$. If $\left|x_{i}(s)\right| / \rho_{i}(s) \leqq 1$ for every $i=1,2, \cdots, n, \sum_{i=1}^{n}\left(\left|x_{i}(s)\right| r_{i} /\left|\rho_{i}(s)\right| r_{i}\right)$ is bounded. If for some $i,\left|x_{i}(s)\right| / \rho_{i}(s) \geqq 1$, then

$$
\sum_{i=1}^{n} \frac{\left|x_{i}(s)\right|^{r_{i}}}{\left[\rho_{i}(s)\right]^{r_{i}}} \leqq n\left(\sum_{i=1}^{n} \frac{\left|x_{i}(s)\right|}{\rho_{i}(s)}\right)^{r}
$$

by Lemma 3, hence 


$$
\sum_{i=1}^{n} \frac{\left|x_{i}(t)\right|}{\rho_{i}(t)} \leqq M+M \int_{0}^{t}\left(\sum_{r j} \epsilon_{r j}(s)\left[\rho_{j}(s)\right]^{r_{i}} \rho_{r}(s)^{-1}\right)\left(\sum_{i=1}^{n} \frac{\left|x_{i}(s)\right|}{\rho_{i}(s)}\right)^{r} d s .
$$

By Lemma 2, $\sum_{i=1}^{n}\left(\left|x_{i}(t)\right| / \rho_{i}(t)\right) \leqq z(t)$ where $z(t)$ is the maximal solution of the differential equation

$$
\dot{z}=M\left(\sum_{r, j} \epsilon_{r j}(s)\left[\rho_{j}(s)\right]^{r i} \rho_{r}(s)^{-1}\right) z^{r}
$$

The solution of (3) is

$$
\begin{aligned}
z(t) & =z(0) \exp M \int_{0}^{t} \sum_{r, j} \epsilon_{r j}(s)\left[\rho_{j}(s)\right]^{r_{j}} \rho_{r}(s)^{-1} d s \quad \text { if } r=1 \\
z(t)^{1-r} & =z(0)^{1-r}+(1-r) \exp M \int_{0}^{t} \sum_{r, j} \epsilon_{r j}(s)\left[\rho_{j}(s)\right]^{r^{i}} \rho_{r}(s)^{-1} d s \text { if } r>1 .
\end{aligned}
$$

Then if $r=1$ all solutions of (3) are bounded in [0, $)$. If $r>1$ choosing $|z(0)| \leqq c$ for $c$ small enough the solutions of (3) are also bounded. Then by Lemma 2 the solutions of (1) exist globally.

Thus in the conditions of hypothesis $\left(\mathrm{H}_{6}\right), x_{i}(t)=h_{i}(t)+\rho_{i}(t) a_{i}(t)$ where

$$
a_{i}(t)=\int_{0}^{t} \sum_{r=1}^{n} \frac{q_{i r}(t-s)}{\rho_{i}(t)} f_{r}(s, x(s)) d s+\int_{0}^{t} \sum_{j=1}^{n} \frac{q_{i j}(t-s)}{\rho_{i}(t)} g_{j}(s) d s
$$

is bounded and in the conditions of hypothesis $\left(\mathrm{H}_{6}^{\prime}\right)$, by Lemma 1 $\lim a_{i}(t)=a_{i}$ constant. The condition of convergence of the integral $\int_{0}^{t} \epsilon_{i j}(t)\left[\rho_{j}(t)\right]^{r_{i}}\left[\rho_{i}(t)\right]^{-1} d t$ in hypothesis $\left(\mathrm{H}_{4}\right)$ cannot be improved as is shown by the simple example

$$
\begin{aligned}
& x_{1}(t)=2 t+1+\int_{0}^{t} 2(t-s)(s+1)^{-2} x_{1}(s) d s, \\
& x_{2}(t)=2+\int_{0}^{t} 2(s+1)^{-2} x_{1}(s) d s
\end{aligned}
$$

which has the solution $\left((t+1)^{2}, 2(t+1)\right)$ which does not satisfy Theorem 1. In this example, $q_{11}=1, q_{12}=t-s, q_{21}=0, q_{22}=1, \epsilon_{11}(t)=0$, $\epsilon_{12}(t)=0, \epsilon_{22}(t)=0, \epsilon_{21}(t)=2(t+1)^{2}, \rho_{1}(t)=2(t+1), \rho_{2}(t)=2$.

The example above shows that Theorem 1 is not true even when $\lim _{t \rightarrow \infty} \epsilon_{i j}(t)=0$. Strauss [8] uses a hypothesis of convergence to zero of the $\epsilon_{i j}(t)$ and obtains similar results but uses a different hypotheses for the kernel $q(t-s)$. 
REMARK 1. It is quite obvious that Theorem 1 is also true when $q=q(t-s)$ is not of convolution type. Hypothesis $\left(\mathrm{H}_{4}\right)$ to $\left(\mathrm{H}_{6}^{\prime}\right)$ were motivated by anterior research on differential equations and are general enough to include implicitly Theorem 2.1 of [3], Theorem 1 of [9] and several results on the boundedness and asymptotic behavior of differential equations, see [2, pp. 37 and 42] for references. The hypotheses imposed to the kernel $q$ can be weakened and are better stated in the context of the more general equation

$$
x(t)=h(t)+\int_{0}^{t} F(t, s, x(s)) d s
$$

where $F=\left(F_{1}, F_{2}, \cdots, F_{n}\right)$ is continuous in $t$ and $x$ and $|F|$ is locally Lebesgue integrable. Theorems on the existence of continuous solutions, continuability of solutions and comparison theorems for (4) can be found in [5], [6] and [7].

We assume also the hypothesis

$$
\begin{aligned}
& \frac{\left|F_{i}(t, s, x(s))\right|}{\rho_{i}(t)} \leqq \sum_{j=1}^{m} g_{i j}(s)\left|x_{j}(s)\right| r_{j}, \\
& \quad i=1,2, \cdots, n, \quad|x|<H \leqq \infty,
\end{aligned}
$$

where $g_{i j}(s)$ is nonnegative, $r_{j} \geqq 0, j=1,2, \cdots, n$, and

$$
\int^{\infty} g_{i j}(t)\left[\rho_{i}(t)\right]^{r_{i}} d t<\infty .
$$

$\left(\mathrm{H}_{7}\right) \lim _{t \rightarrow \infty}\left(F_{i}(t, s, x(s)) / \rho_{i}(t)\right)$ exist and is finite for almost all values of $s$ and $\left(\mathrm{H}_{7}\right)$ is satisfied.

Then conditions $\mathrm{A}$ and $\mathrm{B}$ of Theorem 1 are satisfied for equation (4), that is, the solutions of (4) satisfy $x_{i}(t)=h_{i}(t)+\rho_{i}(t) a_{i}(t)$ with $a_{i}(t)$ bounded in the conditions of hypothesis $\left(\mathrm{H}_{7}\right)$ and $\lim _{t \rightarrow \infty} a_{i}(t)=a_{i}$ in the conditions of hypothesis $\left(H_{7}^{\prime}\right)$. Still more generally let $\rho=\left(\rho_{i}\right)$ be a diagonal matrix, $\rho_{i}(t)>0, i=1,2, \cdots, n$, chosen in such a way that $\left|\rho(t)^{-1} h(t)\right| \leqq K$. Suppose that $\left|\rho(t)^{-1} F(t, \quad s, \quad x(s))\right|$ $\leqq \omega\left(t, s,\left|\rho(s)^{-1} x(s)\right|\right)$ where $\omega(t, s, r)$ is monotone nondecreasing in $r$ for each fixed pair $(t, s)$. Then if the solutions of the integral equation $y(t)=K+\int_{0}^{t} \omega(t, s, y(s)) d s$ are bounded, the solutions of (4) satisfy $x(t)=h(t)+\rho(t) a(t)$ with $a(t)$ bounded. If in addition $\lim _{t \rightarrow \infty} \rho(t)^{-1} F(t, s, x(s))$ exist and is finite then $\lim _{t \rightarrow \infty} a(t)=a$ const.

REMARK 2. The following example shows that without hypothesis $\left(\mathrm{H}_{7}^{\prime}\right)$ we can not really guarantee that $\lim _{t \rightarrow \infty} a_{i}(t)$ exist. Consider the linear integral equation 


$$
\begin{aligned}
x(t)= & +\frac{1}{1+t}+\cos \left(\frac{1}{2}-\frac{1}{1+1 /(1+t)}\right) \\
& -\cos t \int_{0}^{t} \frac{1}{(1+s)^{2}(1+1 /(1+s))^{3}} x(s) d s
\end{aligned}
$$

which has the solution $x(t)=1+1 /(1+t) . \quad h(t)=1+1 /(1+t)-$ $\cos t(1 / 1+1 /(1+t))+\frac{1}{2} \cos t$. We can put $\rho(t)=1+1 /(1+t)$. Then

$$
\begin{aligned}
\frac{x(t)-h(t)}{\rho(t)} & =\frac{\cos t}{1+1 /(1+t)} \int_{0}^{t} \frac{1}{(s+1)^{2}(1+1 /(1+s))^{3}} x(s) d s \\
& =\frac{\cos t}{1+1 /(1+t)}\left[\frac{1}{1+1 /(1+t)}-\frac{1}{2}\right]
\end{aligned}
$$

which does not have a limit when $t \rightarrow \infty$.

In an analogous manner the integral equation

$$
\begin{aligned}
x(t)=1 & +\frac{1}{1+t}-\frac{1}{1+1 /(1+t)}+\frac{1}{2} \\
& -\int_{0}^{t} \frac{1}{(1+s)^{2}(1+1 /(1+s))^{3}} x(s) d s
\end{aligned}
$$

shows that, in general, we can not have

$$
\lim _{t \rightarrow \infty} \frac{(x(t)-h(t))}{\rho(t)}=0 .
$$

REMARK 3. If $h(t)=$ constant in (1), then in the condition of hypothesis $\left(\mathrm{H}_{6}^{\prime}\right), \lim _{t \rightarrow \infty} x(t)=C$ constant if $x(t)$ is bounded in $[0, \infty)$. Also the solutions of (1) tend to a point of equilibrium and equation (1) has asymptotic equilibrium in the sense defined by Wintner for differential equations (see [2, p. 43]).

REMARK 4. As a simple application of Theorem 1 consider the equation

$$
y^{(n)}+f\left(t, y^{(1)}, \cdots, y^{(n-1)}\right)=g(t)
$$

which is equivalent to the integral equation

$$
y^{(k)}(t)=\theta_{0}+\theta_{1} t+\cdots+\theta_{n-k-2} t^{n-k-1}+\int_{t_{0}}^{t} \frac{(t-s)^{n-k-1}}{(n-k-1) !} g(s) d s
$$

$$
\begin{aligned}
&-\int_{t_{0}}^{t} \frac{(t-s)^{n-k-1}}{(n-k-1) !} f\left(s, y^{(1)}(s), \cdots, y^{(n)}(s)\right) d s, \\
& K=0,1, \cdots, n-1 .
\end{aligned}
$$


If we choose $\rho_{k}(t)=t^{n-k-1}$, the kernel of (6) satisfies hypotheses $\left(\mathrm{H}_{1}^{\prime}\right)$ and $\left(\mathrm{H}_{6}^{\prime}\right)$. If $f$ and $g$ satisfy the hypotheses of Theorem 1 then (5) has a solution satisfying $\lim \left(y^{(k)}(t) / t^{n-k-1}\right)=A_{K}$. Since the integrals in (6) are convergent for $t_{0}$ sufficiently large, $A_{K} \neq 0, K=1,2, \cdots$, $n-1$, and we obtain Theorem 2.1 of [2] with weaker hypotheses which contains Theorem 1 of [9]. Brauer and Wong [1] treat the same problem from a different point of view and obtain the same results in a more general context.

Acknowledgments. I thank Dr. R. K. Miller for reading the paper and for helpful discussions. The results of this paper were obtained in the Center for Dynamical Systems while the author held a position as Visiting Assistant Professor.

\section{REFERENCES}

1. F. Brauer and J. S. W. Wong, On asymptotic behavior of perturbed linear systems, J. Differential Equations 6(1969), 142-153. MR 39 \#570.

2. L. Cesari, Asymptotic behavior and stability problems in ordinary differential equations, 2nd ed., Academic Press, New York and Springer-Verlag, Berlin, 1963. MR $27 \# 1661$.

3. T. G. Hallam, Asymptotic behavior of the solutions of an nth order nonhomogeneous ordinary differential equation, Trans. Amer. Math. Soc. 122 (1966), 177-194. MR 32 \#6000.

4. E. W. Hobson, The theory of functions of a real variable and the theory of Fourier series. Vol. 2, Dover, New York, 1958. MR 19, 1166.

5. R. K. Miller, Nonlinear Volterra integral equations, Lecture Note Series, Benjamin, New York (to appear).

6. J. A. Nohel, Some problems in nonlinear Volterra integral equations, Bull. Amer. Mat. Soc. 68 (1962), 323-329. MR 26 \#2838.

7. T. Sato and A. Iwasaki, Sur l'équation intégrale de Volterra, Proc. Japan Acad. 31 (1955), 395-398. MR 17, 751.

8. A. Strauss, On a perturbed Volterra integral equation, Arch. Rational Mech. Anal. (to appear).

9. P. Waltman, On the asymptotic behavior of solutions of a nonlinear equation, Proc. Amer. Math. Soc. 15 (1964), 918-923. MR 31 \#445.

Escola de Engenharia de São Carlos, São Carlos, Brasil

Center for Dynamical Systems, Brown University, Providence, Rhode ISLAND 02912 